(POLARIS-2 and POLARIS-3) patients with and without compensated cirrhosis. DAA-experienced patients received treatment for 12 weeks, and DAA-naive patients received treatment for 8 weeks. Overall SVR12 rates were $>95 \%$ across all the studies. This post-hoc analysis assesses efficacy in patients with and without traditional negative predictors of response.

Methods This was a retrospective analysis of data from 1056 patients treated with SOF/VEL/VOX in the Phase 3 studies. Presence of cirrhosis was determined by histology, Fibrotest/ APRI, or Fibroscan. Viral load and other clinical and laboratory assessments were determined prior to treatment with SOF/VEL/VOX. Prior treatment records were source verified, and race was self-reported by the patient to the investigator.

Results Overall, 38\% of patients had cirrhosis, $70 \%$ had HCV RNA $\geq 800,000 \mathrm{IU} / \mathrm{mL}, 59 \%$ of the DAA-experienced patients had received an NS5A inhibitor-containing regimen, 20\% of the DAA-naive patients had prior treatment failure with pegylated interferon +ribavirin, $12 \%$ were $\geq 65$ years old and $10 \%$ were black.

Conclusions The POLARIS-1, POLARIS-2, POLARIS-3, and POLARIS-4 studies enrolled a diverse patient population that included a significant number of patients with historically negative predictors of response including cirrhosis and prior exposure to DAA-containing regimens. High SVR12 rates for the ribavirin-free regimen of SOF/VEL/VOX were achieved across subgroups.

\begin{tabular}{|c|c|c|}
\hline Overall & 430/445 (97) & $582 / 611$ (95) \\
\hline Cirrhosis & 194/205 (95) & $188 / 200$ (94) \\
\hline HCV RNA $\geq 800 \mathrm{~K}$ & $317 / 326(97)$ & $392 / 416$ (94) \\
\hline Prior PEG+RBV & - & $114 / 124(92)$ \\
\hline Age $>65$ & 73/74 (99) & 55/57 (96) \\
\hline Black & 50/54 (93) & $43 / 48(90)$ \\
\hline
\end{tabular}

\section{IDDF2018-ABS-0120 THE EFFECT OF OMEGA-3 FATTY ACID SUPPLEMENTATION ON PAEDIATRIC NON- ALCOHOLIC FATTY LIVER DISEASE: A META-ANALYSIS OF RANDOMISED CONTROLLED TRIALS}

${ }^{1}$ Martin MH Wong*, ${ }^{2}$ Kenneth Lo, ${ }^{3}$ Wilson Tam. ${ }^{1}$ The Nethersole School of Nursing, The Chinese University of Hong Kong, Hong Kong; ${ }^{2}$ School of Public Health and Primary Care, The Chinese University of Hong Kong, Hong Kong; ${ }^{3}$ Alice Lee Centre for Nursing Studies, Yong Loo Lin School of Medicine, National University of Singapore, Singapore

\subsection{6/gutjint-2018-IDDFabstracts.215}

Background Paediatric non-alcoholic fatty liver disease (NAFLD) is an obesity-related disease with growing prevalence worldwide. While current guidelines recommend lifestyle modification, omega-3 supplementation has been suggested as an emerging therapeutic option. Despite supporting evidence demonstrated among adult NAFLD patients, similar meta-analysis among the paediatric population is lacking. The present review aimed to evaluate the efficacy of omega-3 supplementation among children with NAFLD.

Methods A systematic review of all randomised controlled trials (RCT) on NAFLD patients aged 18 or under was conducted using 16 multiple databases including Medline, EMBASE and Cochrane Central Register of Controlled Trials. PRISMA guideline was followed. The search was conducted by two independent reviewers, with discrepancies resolved by the third reviewer. Outcomes were categorised into anthropometric (e.g. body mass index), cardio-metabolic (e.g. triglycerides and insulin resistance) and hepatic outcomes (e.g. liver enzymes). Using mean differences (MD), meta-analysis was conducted on outcomes reported in more than one study using a random-effect model. If only median and inter-quartile ranges were provided, they were transformed into mean and standard deviation before meta-analysis. Heterogeneity was examined using $\mathrm{I}^{2}$ statistics. The Cochrane Collaboration's tool for assessing the risk of bias was used to examine the quality of the included studies.

Results After screening 2962 papers, six papers with 326 subjects from five RCT were included in the present review. The included studies were conducted in Canada, Italy, Poland and Turkey. Omega-3 supplementation significantly increased body mass index $\mathrm{z}$-score $(\mathrm{MD}=0.09)$, reduced the levels of serum triglycerides $(\mathrm{MD}=-9.52)$ and alanine aminotransferase (ALT) $(\mathrm{MD}=-12.04)$, and improved insulin sensitivity (MD of the homeostasis model assessment of insulin resistance $=-0.49$ ). Heterogeneity was low (below 30\%) across various outcomes, and the study quality was generally high, with three papers having low risks of bias in all six domains.

Conclusions To conclude, omega-3 supplementation reduced serum triglycerides and ALT, and improved insulin sensitivity among children with NAFLD. It may act as a potential supplementation for paediatric NAFLD patients. But more trials should be conducted, particularly in Asian regions.

\section{IDDF2018-ABS-0124 IDENTIFICATION OF MIR-148A IN PLASMA AS A POTENTIAL NONINVASIVE BIOMARKER FOR HEPATOCELLULAR CARCINOMA}

${ }^{1}$ Juqiang Han*, ${ }^{2}$ Jiarui Li, ${ }^{3}$ Wenpeng Liu, ${ }^{2}$ Jiguang Liang, ${ }^{4}$ Zhigang Huang, 'Shuai Wang, ${ }^{3}$ Caiyan Zhao, ${ }^{5}$ Xiaojie Xu, ${ }^{5}$ Qinong Ye. ${ }^{1}$ Department of Liver Disease, PLA Army General Hospital, China; ${ }^{2}$ Department of Interventional Radiography, The First Hospital of Jilin University, China; ${ }^{3}$ Department of Infectious disease, The Third Hospital of Hebei Medical University, China; ${ }^{4}$ Department of Epidemiology, Guangdong Medical University, China; ${ }^{5}$ Department of Medical Molecular Biology, Beijing Institute of Biotechnology, China

\subsection{6/gutjnl-2018-IDDFabstracts.216}

Background Detection of microRNA (miRNA) aberrations in the peripheral plasma is a new approach for hepatocellular carcinoma (HCC) screening. The aim of this study was to characterise miR-148a in the peripheral plasma as a non-invasive biomarker for diagnosis of HCC.

Methods Plasma-based miR148a analysis was performed on 346 plasma samples, including 155 HCCs, 96 liver cirrhosis and 95 healthy controls by using quantitative Real-Time PCR (qRT-PCR). Subsequently, plasma-based miR148a levels were validated in 97 pairs of HCC followed-up after removal of 